\title{
TUNED Research

\section{Efecto del alojamiento de las gallinas (pastoreo, piso y jaula) sobre ácidos grasos, consumo, y percepción sensorial de sus huevos}

\author{
Sianny Chavarría-Zamora ${ }^{1}$ (D); Alejandro Chacón-Villalobos ${ }^{2}$ (D), Rodolfo WingChing-Jones ${ }^{3}$ \\ 1. Universidad de Costa Rica, Escuela de Zootecnia, San José, Costa Rica; sianny.chavarria@ucr.ac.cr \\ 2. Universidad de Costa Rica, Estación Experimental Alfredo Volio Mata, Cartago, Costa Rica; \\ alejandro.chacon@ucr.ac.cr \\ 3. Universidad de Costa Rica, Escuela de Zootecnia, Centro de Investigación en Nutrición Animal, San José, Costa Rica; \\ rodolfo.wingching@ucr.ac.cr
}

Recibido 11-XI-2020 • Corregido 12-I-2021 • Aceptado 25-I-2021

DOI: https://doi.org/10.22458/urj.v13i1.3317

\begin{abstract}
Effect of hen housing (free-range, free cage, and cage) on fatty acids, consumption, and consumer perception of the eggs. Introduction: At a global level, consumer concern is growing for animal welfare, including hen housing in the production of eggs. Objectives: To assess the effect of hen housing conditions on some egg characteristics. Methods: The study was done in Costa Rica. We made a standard fatty acid profile analysis of 30 eggs, randomly selected from each housing system. We also surveyed 777 habitual egg consumers about their purchase and consumption habits, and applied a sensory test to 108 panelists. Results: The polyunsaturated fatty acids / saturated fatty acids ratios were $0.58,0.55$ and 0.51 for the free-range, free cage and cage system, respectively. In the sensory panel, $40 \%$ of the panelists were grouped for their greater general liking towards the sample, with preference for the free-range eggs. Slightly over half ( $55 \%$ ) of the sampled population was unaware of the egg production systems. Conclusion: The acid ratio of these Costa Rican eggs is labeled as "healthy food" and nearly half of the surveyed population is unaware of the egg production systems.
\end{abstract}

Keywords: eggs, housing systems, purchase, consumption, trends.
RESUMEN. Introducción: A nivel mundial, crece la preocupación de los consumidores por el bienestar de los animales, incluidas las gallinas en el caso de los huevos. Objetivos: Evaluar el efecto de las condiciones de alojamiento de las gallinas sobre algunas características del huevo. Métodos: El estudio se realizó en Costa Rica. Hicimos un análisis estándar de perfil de ácidos grasos de 30 huevos, seleccionados al azar para cada uno de los sistemas de alojamiento. También encuestamos a 777 consumidores habituales de huevos sobre sus hábitos de compra y consumo y aplicamos una prueba sensorial a 108 panelistas. Resultados: Las proporciones de ácidos grasos poliinsaturados / ácidos grasos saturados fueron de 0,58, 0,55 y 0,51 para pastoreo, piso y jaula, respectivamente. En el panel sensorial, el $40 \%$ de los panelistas se agruparon por su mayor agrado general hacia la muestra, con preferencia por los huevos de gallinas de pastoreo. Poco más de la mitad (55\%) de la población muestreada desconocía los sistemas de producción de huevos. Conclusión: La proporción de ácidos de estos huevos costarricenses los califica como "alimento saludable" y casi la mitad de la población encuestada desconoce los sistemas de producción de huevos.

Palabras clave: huevo, alojamiento para aves, compra, consumo, tendencias. 
El huevo es un alimento que se caracteriza por su versatilidad culinaria, bajo costo, bajo contenido de calorías, proteína de alto valor biológico (Carrillo, 2013), y es fuente de ácidos grasos, lípidos y colesterol necesarios en la dieta de los seres humanos (Holt et al., 2010). Sobresale de otros alimentos de origen proteico debido a que contiene todas las vitaminas a excepción de la vitamina C, y minerales como el hierro, fósforo, zinc, selenio y yodo (Tortuero, 2002; Carbajal, 2006; Rodríguez, 2016).

Además, presenta ácidos grasos esenciales para el ser humano como el oleico, linoleico (omega 6), linolénico (omega 3) (Carbajal, 2006), ácido eicosapentaenoico (EPA) y ácido docosahexaenoico (DHA), los cuales disminuyen el riesgo de enfermedades cardiovasculares, diabetes y desórdenes neurológicos (Jing, Zhao, \& House, 2017). Otros lípidos como la lecitina que está involucrada en el desarrollo normal del cerebro, y actúa como precursor en el desarrollo de la memoria y el aprendizaje (Instituto de Estudios del Huevo, 2009). Pigmentos como la luteína y la zeaxantina tienen función antioxidante, antimutagénica y anticancerígena (Mares-Perlman, Millen, Ficek, \& Hankinson, 2002; Ribaya-Mercado \& Blumberg, 2004).

Dentro de los factores que pueden afectar la calidad sensorial del huevo se encuentran la cantidad de ácidos grasos poliinsaturados, debido a que estos pueden sufrir procesos de oxidación, la edad del ave, el tiempo de almacenamiento del huevo y el sistema de alojamiento (Karsten, Patterson, Stout \& Crews, 2010; Rakonjac et al., 2014), donde este último factor supone diferencias en cuanto al manejo del ave, la alimentación e inocuidad del huevo (Hidalgo, Rossi, Clerici \& Ratti, 2008).

En cuanto a consumo de huevo a nivel mundial se destaca que, debido al crecimiento poblacional, para el año 2030 se proyecta un consumo per cápita de 8,9kg de huevo en países de vías de desarrollo, mientras que en los países más desarrollados se espera un consumo que alcance los 13,8kg de huevo por persona al año (Conway, 2012). En Costa Rica, desde el año 2007 al año 2016, el consumo per cápita de huevo en su mayoría en color marrón tuvo un aumento de 3,5 unidades anuales, alcanzando un consumo de 13,70kg de huevo por persona al año (Gutiérrez, 2017).

A partir del 2000 ha crecido la preocupación de los consumidores de huevo en la Unión Europea en torno al bienestar animal de las aves (Nocella, Hubbar, \& Scarpa, 2009), ya que el 77\% consideran como necesario realizar mejoras entorno al bienestar animal en granjas avícolas (European Commission, 2007), debido a que desean consumir productos que sean saludables, amigables con el medio ambiente y que respeten los lineamientos del bienestar animal (Villanueva et al., 2015).

Un comportamiento similar a los consumidores europeos se puede apreciar en los Estados Unidos, donde se percibe la necesidad al adquirir los productos de informarse de la procedencia, manejo de los productos que se consumen, así como el bienestar animal y sustentabilidad ambiental bajo la cual son producidos (Ellison, Brooks \& Mieno, 2017), e incluso los compradores han indicado sentirse dispuestos a pagar más por este tipo de productos (Heng, Peterson, \& Li, 2013). Además de las crecientes consternaciones individuales, grandes empresas internacionales dedicadas a la preparación de comidas rápidas informaron que solo comprarán huevos provenientes de sistemas libres de jaula, para ser utilizados en sus platillos (Strom, 2015).

En Costa Rica se desconoce si los consumidores tienen estas consternaciones e intenciones de compra en torno al huevo de gallina, por lo que esta investigación tuvo como objetivos la caracterización del perfil de ácidos grasos del huevo, establecer por medio de una encuesta los hábitos e intenciones de compra del consumidor costarricense y realizar un panel sensorial de agrado de huevo proveniente de tres sistemas de alojamiento diferentes (sistema convencional de jaula, piso y con acceso a pastoreo). 


\section{MATERIALES Y MÉTODOS}

Generalidades: La investigación consistió en un estudio descriptivo del perfil de ácidos grasos y agrado sensorial del huevo proveniente de gallinas de la línea genética Hy-Line Brown (HLB) (jaula), Novogen (NG) (piso), de la raza Rhode Island Red (RIR) y el cruce Sex Link (SL) (con acceso a pastoreo).

Características de los sistemas productivos: El estudio se realizó entre los meses de diciembre del año 2017 y mayo del 2018, con huevos provenientes de las gallinas en tres sistemas de alojamiento (jaula, piso y con acceso a pastoreo) ubicados en el cantón central de Turrialba,

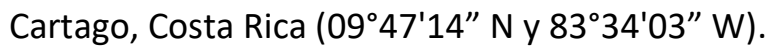

Procedimientos evaluados: Se realizó un análisis de perfil de ácidos grasos, un panel sensorial de los huevos provenientes de cada genética de las aves, y una encuesta a 794 personas, de las cuales 777 consumidores indicaron ser consumidores habituales de huevo, informaron sobre sus hábitos de compra y consumo del huevo de gallina.

Para la caracterización del perfil de ácidos grasos, se tomó una muestra al azar de 30 unidades de huevo de cada sistema de alojamiento (jaula, piso y con acceso a pastoreo), estos se colocaron en transportadores plásticos de huevo los cuales a su vez se depositaron en cajas plásticas y fueron llevados al laboratorio del Centro de Investigación en Nutrición Animal (CINA) de la Universidad de Costa Rica, para el análisis del perfil de ácidos grasos basado en la metodología descrita por Misir, Laarveld, \& Blair (1985).

En cuanto al análisis sensorial, se llevó a cabo un panel sensorial con consumidores de huevo en el cual se evaluó el agrado general del huevo utilizando como herramienta la escala híbrida, siguiendo la metodología de Villanueva, Petenate, \& Da Silva (2005). Las muestras para dicho panel se prepararon y presentaron según lo descrito por Zamora (2013), lo cual consistió en tortas de huevo de $3 \mathrm{~cm}$ de diámetro las cuales se manejaron en igualdad de condiciones, provenientes de huevos que tenían un día de haber sido puestos y almacenados a temperatura ambiente $\left(25^{\circ} \mathrm{C}\right)$.

El panel sensorial tuvo lugar en el laboratorio de la Escuela de Tecnología de Alimentos de la Universidad de Costa Rica, abarcando una muestra de 108 consumidores habituales de huevo. A cada panelista se le presentaron tres tortas pertenecientes a cada sistema de alojamiento y genéticas correspondientes, a las cuales, se les asignó una numeración al azar de tres dígitos con la finalidad de que la persona no supiera a cuál muestra correspondía determinado alojamiento, además el orden de presentación de la numeración varió al azar.

Cada participante recibió previamente instrucciones de cómo desarrollar la actividad, y se le ubicó en un cubículo con una computadora que mostraba las escalas hibridas no estructuradas según lo recomendado por Villanueva et al. (2005) de $10 \mathrm{~cm}$ de longitud para establecer el agrado que iba de $0 \mathrm{~cm}$ : desagradable, $5 \mathrm{~cm}$ : indiferente a $10 \mathrm{~cm}$ : agradable. Cuando el panelista terminaba, se recogía la muestra y el resultado quedaba registrado en una hoja de cálculo del software Microsoft Excel. Con los datos obtenidos para las medias de todos los datos en general, se realizó un ANOVA, al no encontrarse diferencias significativas $(p>0,05)$ en cuanto al agrado, se realizó un análisis de conglomerados por medio de la técnica Ward. Una vez anidados en conglomerados se realizó el análisis de varianza con el programa JMP SAS versión 4.0 a cada grupo obtenido para determinar diferencias significativas entre medias usando la prueba de Tukey.

El objetivo de realizar el panel sensorial de agrado general consistió en establecer si los panelistas (consumidores habituales de huevo) podían percibir diferencias en las muestras provenientes de los tres sistemas de alojamiento y sus respectivas genéticas.

Por último, se realizó una encuesta por medio de la plataforma Google Forms con un total de 18 preguntas, divididas según hábitos de compra, hábitos de consumo y conocimiento sobre los 
sistemas de producción de huevo a 777 consumidores habituales de huevo en Costa Rica, con el fin de establecer si el comprador tiene conocimiento sobre la existencia de diferentes sistemas de producción de huevo, si percibe diferencias entre estos y si está dispuesto o no pagar un sobreprecio por las mismas, así como establecer de forma descriptiva sus hábitos de consumo y compra del huevo. Los datos se analizaron por medio de Excel de forma descriptiva.

\section{RESULTADOS}

Perfil de ácidos grasos: Al realizar el análisis del perfil de ácidos grasos en las muestras de huevo fresco provenientes de gallinas de diferentes genéticas en tres sistemas de alojamiento (jaula, piso y con acceso a pastoreo) se identificaron entre 12 y 15 ácidos grasos en total, de los cuales el oleico $(46,85 \pm 0,85)$, palmítico $(24,20 \pm 1,30)$ y linoleico $(14,55 \pm 1,28)$ son los que presentaron una mayor proporción (Tabla 1). De forma general, el ácido oleico presenta una relación con el palmítico y el linoleico determinada en este trabajo de 1,93:1 y 3,22:1 respectivamente. Mientras que; 1,66:1 refleja la relación entre ácido palmítico y linoleico en el huevo. Por último, la relación entre ácidos grasos poliinsaturados y monoinsaturados encontrada en este trabajo según el tipo de alojamiento de las aves es de 0,33, 0,32 y 0,38 para las aves HBL en jaula, NG en piso y RIR+SL con acceso a pastoreo. En el caso de ácidos saturados y poliinsaturados se obtienen relaciones de 0,55, 0,51 y 0,58 respectivamente.

TABLA 1

Perfil de ácidos grasos de la yema de huevo según sistema de alojamiento

\begin{tabular}{lccc}
\hline \multirow{2}{*}{ Compuesto } & \multicolumn{2}{c}{ Suma relativa de áreas (g/100g) } \\
\cline { 2 - 4 } & Jaula & Piso & Pastoreo \\
\hline Ácido 6-oxa-3tiooctanóico & 0,38 & $\mathrm{ND}$ & $\mathrm{ND}$ \\
Ácido benzoico & 2,28 & 0,87 & 1,40 \\
Ácido tetradecanóico (mirístico) & 0,52 & $\mathrm{ND}$ & 0,39 \\
Ácido 2-metilhetanóico & 0,06 & $\mathrm{ND}$ & $\mathrm{ND}$ \\
Ácido hexadecanóico (pamítico) & 22,85 & 25,46 & 24,30 \\
Ácido 11-metilhexadecanóico, (Z-) & 4,19 & $\mathrm{ND}$ & 3,57 \\
Ácido 9-metilhexadecanóico, (Z-) & $\mathrm{ND}$ & 4,86 & $\mathrm{ND}$ \\
Ácido 2,4-di-t-butilfenil-5-hidroxipentanóico & 0,08 & 0,12 & $\mathrm{ND}$ \\
Ácido tridecanóico & 0,12 & 0,42 & $\mathrm{ND}$ \\
Ácido undecanóico & $-\mathrm{ND}$ & 0,08 & $\mathrm{ND}$ \\
Ácido 10-undecanóico & 0,11 & $\mathrm{ND}$ & $\mathrm{ND}$ \\
Ácido 15-metilhexadecanóico & $\mathrm{ND}$ & 0,09 & 0,13 \\
Ácido 9-dodecanóico, (Z-) & $\mathrm{ND}$ & 0,13 & 0,12 \\
Ácido octadecanóico (esteárico) & 7,43 & 5,74 & 7,37 \\
Ácido (Z)-9-octadecenóico (oleico) & 47,01 & 46,34 & 45,32 \\
Ácido (Z-Z)-9,12-octadecenóico (linoléico) & 13,48 & 14,20 & 15,98 \\
Ácido 11,14-ecosadienóico & 0,64 & 0,20 & 0,36 \\
Ácido11,14,17-eicosatrienóico & $\mathrm{ND}$ & 0,22 & 0,41 \\
Ácido 3,5-bis(1,1-dimetiletil)-4-hidroxi-bencenpropanóico & $\mathrm{ND}$ & 0,43 & 0,66 \\
Ácido (Z-Z)-9,15-octadecenóico & 0,49 & $\mathrm{ND}$ & $\mathrm{ND}$ \\
Ácido trans-2-(3-ciclopropil-7-norcaranil) acético & 0,37 & $\mathrm{ND}$ & $\mathrm{ND}$ \\
Ácido araquidiónico & $\mathrm{ND}$ & 0,35 & $\mathrm{ND}$ \\
Suma de ácidos grasos saturados (AGS) & $\mathbf{3 1 , 3 6}$ & $\mathbf{3 1 , 9 1}$ & $\mathbf{3 2 , 1 9}$ \\
Suma de ácidos grasos monoinsaturados (AGMI) & $\mathbf{5 1 , 3 1}$ & $\mathbf{5 1 , 7 0}$ & $\mathbf{4 9 , 0 1}$ \\
Suma de ácidos grasos poliinsaturados (AGPI) & $\mathbf{1 7 , 3 4}$ & $\mathbf{1 6 , 3 9}$ & $\mathbf{1 8 , 8 1}$ \\
\hline ND & & &
\end{tabular}

$\mathrm{ND}=$ no detectado. 
Análisis sensorial: Se agruparon los conglomerados que forman menos aumentos en la suma de las distancias dentro de cada conglomerado (De la Fuente, 2011; Fig. 1) y se encontró que los conglomerados muestran diferencias en las tendencias de agrado.

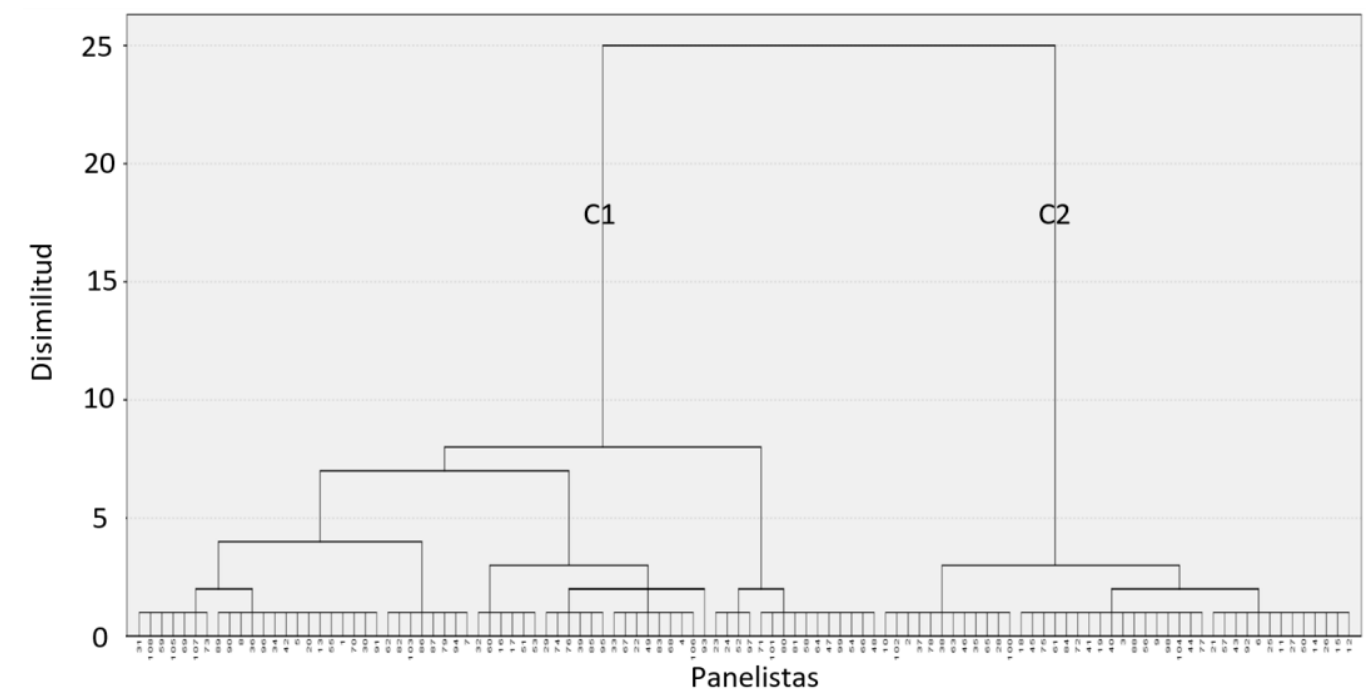

Fig. 1. Dendograma de conglomerados obtenidos por la técnica de Ward en función de agrado del huevo.

El conglomerado 1 (C1) agrupó al 61,1\% (66 personas) de los panelistas, los cuales exhibieron menor agrado general por las tortas de huevo, mientras que en el conglomerado 2 se aglomeró el restante $38,9 \%$ de los panelistas (33 personas) los cuales mostraron un mayor agrado general hacia la muestra (Tabla 2).

\section{TABLA 2}

Diferencias de media del agrado general de tortas de huevo, por conglomerados según sistema de alojamiento y genética de las aves

\begin{tabular}{ccc}
$\begin{array}{c}\text { Alojamiento y genética de las } \\
\text { aves }\end{array}$ & Conglomerado 1 & Conglomerado 2 \\
\hline Acceso a pastoreo (RIR + SL) & $6,194^{\mathrm{c}}$ & $9,293^{\mathrm{a}}$ \\
Jaula (HLB) & $6,638^{\mathrm{b}}$ & $8,648^{\mathrm{b}}$ \\
Piso (NG) & $7,183^{\mathrm{a}}$ & $8,843^{\mathrm{b}}$ \\
\hline b y c= letras diferentes en la misma columna indican que hay diferencias significativas $(\mathrm{p}<0,05)$
\end{tabular}

El conglomerado 1 presentó diferencias significativas entre los sistemas, siendo las tortas de huevos provenientes del sistema de piso y la línea NG el que mostró un mayor agrado. En el caso del conglomerado 2 , también hubo diferencias significativas $(p<0,05)$, mostrando un mayor agrado por las muestras procedentes del sistema con acceso a pastoreo de las gallinas RIR y SL, mientras que entre las muestras provenientes las gallinas HLB (jaula) y NG (piso) no se encontraron diferencias significativas.

Hábitos de compra y consumo del huevo: El 97,9\% (777 personas) indicaron consumir huevo de forma frecuente (Fig. 2). De la población total el 69,3\% corresponde a mujeres y el otro $30,7 \%$ a hombres, con un grado de escolaridad superior a la educación secundaria concluida del $81,9 \%$. 


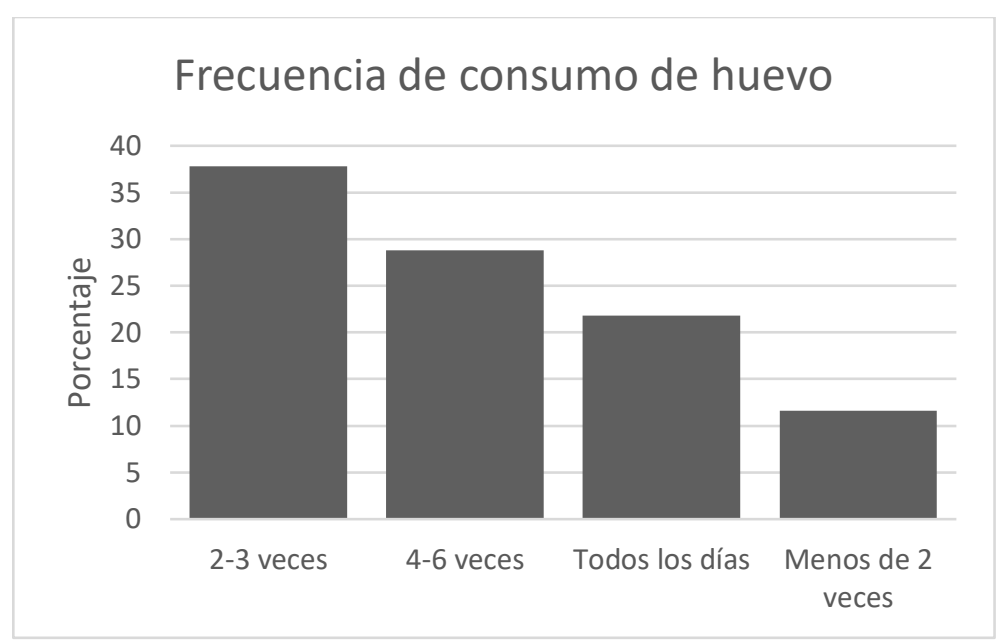

Fig. 2. Frecuencia de consumo de huevo por semana en los encuestados costarricenses.

El 96,7\%, consideró que el huevo es un alimento sano, el cual consumen porque lo consideran como nutritivo, de buen sabor y de fácil preparación. Del total de los encuestados, solo el 2,1\% (17 personas) señaló no consumir huevo, debido a el desagrado por el mismo (44,4\%), seguido porque llevan a cabo prácticas como el veganismo $(38,9 \%)$ y por razones de salud $(16,7 \%)$. Los que indicaron que no consumen huevo por cuestiones de salud representan un $0,37 \%$ del total de los participantes de la encuesta.

El 73,1\% de los encuestados indicó como lugares predilectos de compra el supermercado, las pulperías o el comercio informal. El cartón de huevo fue la presentación predilecta de compra $(72,0 \%)$ en comparación con adquirirlo al menudeo (9,2\%). Los compradores señalaron que al momento de adquirir el producto no se fijan en si el huevo es casero, de pastoreo o enriquecido, y señalaron como características más importantes el tamaño del huevo y su precio, seguido por la limpieza y frescura de este. Además, dentro del análisis se determinó que el comprador le da poco énfasis al sabor y al empaque.

En cuanto al conocimiento sobre los sistemas de producción de huevo, el 44,5\% señaló tener algún conocimiento sobre los mismos; al dar una breve descripción se encontraron respuestas como "hacinadas, poco espacio", "viven en jaulas o en corrales". Mientras que el restante 55,5\% de los encuestados exteriorizaron desconocer por completo las condiciones de producción.

El 70,4\% del total de los consumidores indicaron como criterios por los cuales pagarían más al comprar el huevo el bienestar animal (desde una concepción propia), seguido por la buena alimentación de las aves y la higiene del producto. 


\section{DISCUSIÓN}

Los ácidos grasos son de importancia ya que varios de ellos como el omega 6, el omega 3, el DHA, el EPA, entre otros no pueden ser sintetizados por el ser humano y se consideran como esenciales (Rodríguez, 2016). En este estudio las aves de los tres sistemas de alojamiento eran de diferentes genéticas, además tuvieron diferentes manejos asociados a los mismos, sumando la sensibilidad de la metodología usada en el laboratorio no es posible comparar entre sí los sistemas y genéticas en cuanto a ácidos grasos saturados (AGS), ácidos grasos monoinsaturados (AGMI), ni ácidos grasos poliinsaturados (AGPI), por lo que se realizó una descripción de estos. Los perfiles de ácidos grasos definidos coinciden en términos porcentuales con el descrito por el Instituto de Estudios del Huevo (2009).

Una relación AGPI/AGS superior a 0,35 hace que un alimento se considere como saludable (Pontes \& Castelló, 1995). Esta relación permite clasificar al huevo de las gallinas RIR y SL con acceso a pastoreo como el que presenta una mejor relación AGPI/AGS lo cual favorece la salud del consumidor (0,58); sin embargo, las diferencias entre las relaciones de las aves NG y HLB no son grandes y también se clasificaron como un alimento saludable ya que la relación AGPI/AGS fue de 0,55 y de 0,51 para el sistema de piso y el sistema de jaula respectivamente.

En la literatura se señala que las diferencias en las características sensoriales de los huevos de gallinas con acceso a pastoreo en comparación con los huevos provenientes de sistemas en jaula se deben a la vegetación ofrecida en los sistemas con acceso a pastoreo (Nys et al., 2011) sin embargo, en este estudio es solo uno de los factores determinantes, ya que pudieron haber influido otros como la genética, el manejo sanitario, la crianza y la alimentación de las aves.

El consumo per cápita de huevo en Costa Rica es de 215 unidades al año (Gutiérrez, 2017). Los costarricenses lo consideran como un producto de fácil preparación y de precio accesible con beneficios nutricionales como proteína de calidad y presencia de vitaminas, que además trae consigo beneficios para la salud (Peña, Castro, \& Martínez, 2011), lo cual coincide con lo encontrado en este estudio ya que los encuestados $(96,7 \%)$ consideran al huevo como un alimento sano, de buen sabor y fácil preparación.

Dentro de la población de esta investigación la concepción que relaciona al huevo con problemas en la salud es poco significativa. En una encuesta realizada a 2813 estadounidenses, señalan que solo el 3,5\% de los encuestados no consumen huevo, además encontraron que el factor que limita la compra del huevo es el precio ( $42 \%$ de los casos) (Ochs, Wolf, Widmanr, \& Bir 2018). Además, estos mismos investigadores, indicaron que los encuestados que tenían conocimiento sobre los sistemas de alojamiento de las aves, no contaban con criterio suficiente para discernir entre los aspectos adversos que pueden tener los sistemas alternativos como mayores tasas de mortalidad, incidencia de canibalismo y problemas de inocuidad.

En otro estudio realizado a 1049 compradores de huevo, con el objetivo de visualizar las consternaciones entorno al bienestar animal de los animales en granjas, se encontraron un comportamiento similar al que reflejan los encuestados con tendencia a pagar más por el huevo, ya que el $85 \%$ de las personas encuestadas en los Estados Unidos, indicaron estar dispuestas a pagar más siempre y cuando se cumpla con los atributos de bienestar animal, incluyendo el acceso a áreas de pastoreo, alojamiento libre de jaulas y que no se practique la muda forzada (Heng et al., 2013).

Una de las problemáticas entre productores de huevo, es que estos no consideran que sus clientes estén dispuestos a pagar más por el producto proveniente de granjas que cumplan con el bienestar animal y esto se deriva del desconocimiento que tienen los consumidores sobre los conceptos de bienestar animal (Nocella et al., 2009). Aunado a esto este estudio halló que el 20,1\% de los compradores consideró como injusto el precio que paga por el huevo.

Muchos señalan la necesidad del bienestar animal pero cuando se trata de precio este es 
uno de los factores más importantes al momento de hacer la compra, por lo tanto, cabe dudar si esta disposición que dicen tener a pagar más huevos producidos bajo estándares de bienestar animal es real.

Teniendo en cuenta las percepciones, tendencias y opiniones de los consumidores, los productores pueden tomar decisiones acertadas basadas en información científica para realizar mejoras en sus sistemas que les permitan continuar con sus negocios.

Se considera que el huevo es un alimento saludable, debido a su buena relación AGPI/AGS, a pesar de diferencias en los alojamientos de las aves, genética y alimentación.

En el conglomerado 2 que mostró mayores niveles de agrado por las tortas de huevo, se encontraron diferencias significativas en cuanto a las muestras provenientes de diferentes genéticas y de sistemas de alojamiento, siendo las muestras del sistema con acceso a pastoreo de las gallinas RIR y SL las más agradables para estos consumidores.

Los resultados evidencian que entre consumidores habituales de huevo aspectos como el sabor, contenido nutricional, precio, tamaño y facilidad de preparación, son los principales factores que se consideran al momento de la compra. Así mismo, las personas se mostraron conscientes de la importancia del bienestar animal de las aves, dada la anuencia mostrada a pagar un sobreprecio por el huevo si este tipo de buen manejo se garantiza.

\section{AGRADECIMIENTOS}

Agradecemos el apoyo del módulo aviar de la Sede Atlántico de la Universidad de Costa Rica, las empresas que colaboraron en el proceso, el Centro de Investigación en Nutrición Animal (CINA), a la Escuela de Tecnología de Alimentos y a todos los participantes del panel sensorial y de la encuesta.

\section{ÉTICA, CONFLICTO DE INTERESES Y DECLARACIÓN DE FINANCIAMIENTO}

Los autores declaramos haber cumplido con todos los requisitos éticos y legales pertinentes, tanto durante el estudio como en el manuscrito; que no hay conflictos de interés de ningún tipo, y que todas las fuentes financieras se detallan plena y claramente en la sección de agradecimientos. Asimismo, están de acuerdo con la versión editada final del documento. El respectivo documento legal firmado se encuentra en los archivos de la revista.

La declaración de aporte de cada autor al manuscrito es la siguiente: S.C.Z.: Recogida y análisis de datos, y redacción. A.C.V.: Análisis de datos, redacción y revisión del manuscrito. R.W.C.J.: redacción y revisión del manuscrito. 


\section{REFERENCIAS}

Carbajal, A. (2006). Calidad nutricional de los huevos y relación con la salud. Revista de Nutrición Práctica, 10(1), 73-76.

Carrillo, W. (2013). Lisozima: Actividad Antibacteriana y Alergenicidad. Actualización en Nutrición, 14(1), $314-326$.

Conway, A. 2012. The Statistical Reference for Poultry Executives. Recuperado de https://www.wattagnet.com/ext/resources/uploadedFiles/WattAgNet/Footer/Footer_Content/2011_Poultry_ Trends.pdf

De la Fuente, S. (2011). Análisis de conglomerados. Facultad de Ciencias Económicas y Empresariales. Universidad Autónoma de Madrid. Recuperado de http://www.fuenterrebollo.com/Economicas/ECONOMETRIA/SEGMENTACION/CONGLOMERADOS/conglomer ados.pdf

Ellison, B., Brooks, K., \& Mieno, T. (2017). Which livestock production claims matter most to consumers? Agriculture and Human Values, 34(4), 819-831. DOI:10.1007/s10460-017-9777-9

European Commission (2007). Attitudes of EU citizens towards Animal Welfare, Special Eurobarometer 270. Brussels, Belgium.

Gutiérrez, M.D.L.A. (13 de octubre de 2017,). Costa Rica: Conmemora "Día Mundial del Huevo". Recuperado de https://avicultura.info/costa-rica-conmemora-dia-mundial-del-huevo/

Heng, Y., Peterson, H. H., \& Li, X. (2013). Consumer attitudes toward farm-animal welfare: The case of laying hens. Journal of Agricultural and Resource Economics, 38(3), 418-434.

Hidalgo, A., Rossi, M., Clerici, F., \& Ratti, S. (2008). A market study on the quality characteristics of eggs from different housing systems. Food Chemistry, 106(3), 1031-1038. DOI: 10.1016/j.foodchem.2007.07.19

Holt, P.S., Davies, R.H., Dewulf, J., Gast, K., Huwe, J.K., Jones, D.R., Waltman, D., \& Willian, K.R. (2010). The Impact of different housing systems on egg safety and quality. Poultry Science, 90(1), 251-262. DOI: 10.3382/ps.201000794

Instituto de Estudios del Huevo (IEH). (2009). El gran libro del huevo. España: Evergráficas, SL.

Jing, M., Zhao, S., \& House, J.D. (2017). Performance and tissue fatty acid profile of broiler chickens and laying hens fed hemp oil and HempOmega ${ }^{\mathrm{TM}}$. Poultry Science, 96(6), 1809-1819. DOI:10.3382/ps/pew476

Karsten, H.D., Patterson, P.H., Stout, R., \& Crews, G. (2010). Vitamins A, E and fatty acid composition of the eggs of caged hens and pastured hens. Renewable Agriculture and Food Systems, 25(1), 45-54. DOI: $10.1017 /$ S1742170509990214

Mares-Perlman, J.A., Millen, A.E., Ficek, T., \& Hankinson, S.E. (2002). The body of evidence to support a protective role of lutein and zeaxanthin in delaying chronic diseases. Overview. The Journal of Nutrition, 132(3), 2065-2070. DOI: $10.1093 / \mathrm{jn} / 132.3 .518 \mathrm{~s}$

Misir, R., Laarveld, B., \& Blair, R. (1985). Evaluation of a rapid method for preparation of fatty acid methyl esters for análisis by gas-liquid chromatography. Journal of Chromatography A, 331(1), 141-148. DOI:10.1016/00219673(85)80015-7

Nocella, G., Hubbard, L., \& Scarpa, R. (2009). Farm Animal Welfare, Consumer Willingness to Pay, and Trust: Results of a Cross-National Survey. Applied Economic Perspectives and Policy, 32(1), 275-297. DOI: 10.1093/aepp/ppp009

Nys, Y., Hincke, M.T., Hernández, A., Rodríguez, A.B., Gómez, J., Jonchère, V., García, J., \& Gautron, J. (2010). Structure, propriétés et minéralisation de la coquille de l'oeuf: rôle de la matrice organique dans le contrôle de la fabrication. Productions Animales, 23(2),143-154. 
Ochs, D.S., Wolf, C.A., Widmanr, N.J.O., \& Bir, C. (2018). Consumer perceptions of egg-laying hen housing systems. Poultry Science, 97(10), 3390-3396. DOI:10.3382/ps/pey205

Peña, M., Castro, A., \& Martínez, T. (2011). Conocimientos, opiniones y prácticas respecto al huevo de gallina en familias de comunidades urbana-rural, Costa Rica. Revista Costarricense de Salud Pública, 20(1), 32-39.

Pontes, M., \& Castelló, J.A. (1995). Alimentación de las Aves. España: Real Escuela de Avicultura.

Rakonjac, S., Bogosavljević-Bošković, S., Pavlovski, Z., Škrbić, Z., Dosković, V., Petrović, M., \& Petričević, V. (2014). Laying hen rearing systems: A review of major production results and egg quality traits. World's Poultry Science Journal, 70(1), 93-104. DOI:10.1017/S0043933914000087

Ribaya-Mercado, J.D., \& Blumberg, J.B. (2004). Lutein and zeaxanthin and their potential roles in disease prevention. Journal of the American College of Nutrition, 23(6), 567S-587S. DOI:10.1080/07315724.2004.1079427

Rodríguez, A. (2016). Tipificación de la calidad del huevo de gallina ecológico y convencional (Tesis doctoral). Universidad Politécnica de Valencia, España.

Strom, S. (2015). McDonald's plans a shift to eggs from only cage-free hens. New York Times. Recuperado de https://www.nytimes.com/2015/09/10/business/mcdonalds-to-use-eggs-from-only-cage-free-hens.html

Tortuero, F. (2002). El huevo en la nutrición y la salud. En A.S. Gallego, R.M. Sastre, F. Tortuero, D.G. Suárez, D.G. Vergara, \& G. López (eds.). Lecciones sobre el Huevo (pp. 145-154). España: Instituto de Estudios del Huevo.

Villanueva, C., Oliva, A., Torres, A., Rosales, M., Moscoso, M., \& Gonzáles, E. (2015). Manual de Producción y Manejo de Aves de Patio. Costa Rica: Centro Agronómico Tropical de Investigación y Enseñanza (CATIE).

Villanueva, N., Petenate, A., \& Da Silva, M. (2005). Performance of the hybrid hedonic scale as compared to the traditional hedonic, self-adjusting and ranking scales. Food Quality and Preference, 16(1), 691-703. DOI:10.1016/j.foodqual.2005.03.013

Zamora, L.M. (2013). Evaluación en la inclusión de harina de camarón en la alimentación de gallinas ponedoras comerciales y su efecto en el desempeño productivo de las aves y las características del huevo. (Tesis inédita de Licenciatura). Universidad de Costa Rica, San José, Costa Rica. 